\title{
How to improve the doctors' humanistic quality
}

\author{
Jinguo Wang ${ }^{1, a}$ and Na Wang ${ }^{2, b^{*}}$ \\ ${ }^{1}$ the First Hospital of Jilin University, Changchun 130021, China; \\ 2 the First Hospital of Jilin University, Changchun 130021, China \\ awangjinguolily@163.com, bwangna080613@163.com
}

Keywords: Doctors humanistic quality, education, system construction.

\begin{abstract}
This paper applies the method of comparative analysis, combining with different theories in our country and at abroad, to discuss the connotation and characteristics of the doctors attentive duties. We adopt the method of economic analysis, apply the han DE formula to the attentive obligation standard and the professional standards, and according to the specific content of medical treatment activity provide different types of measures of the doctors attentive duties.
\end{abstract}

\section{Introduction}

In the aspect of medical dispute resolution, doctors duty of care provides objective judgment standard. The damage behavior of legislative no specific provisions, the law could be determined according to the connotation of the attention obligation to undertake responsibility. In the case of physicians attention obligation, breach of duty of care requirements, causing damage of patients, the law can be concluded that medical institutions bear legal responsibility. The stronger the applicability of the law.

In promoting medical technology development, constantly learning new medical physician duty of care requires knowledge, improving medical technology, medical skills, encourage good innovative treatment, allowing for a reasonable risk [1]. In patients with medical technology limitations and special physical fitness and other special cases, doctors can relieve responsibility, in order to fully exercise the right to make a diagnosis and give treatment arbitrary. Duty of care to protect the interests of the patients, doctors can make people understand the specific content of the medical behavior, effective supervision of medical service quality, implement law protect the patient's health and safety of the important goals of life.

\section{Text}

In recent years, with sustained and rapid economic development, people's requirement for the quality of medical and health services is increasing, not simply meet the purpose of treatment, patients also are very concerned about the medical quality and service attitude. Existing health resources and service content is difficult to meet the needs of the masses of the people, so the conflicts and shield.

The specific measure of doctors duty of care is "medical judgment rule", "respect for minority rule", "best judgment law", "allow risk law", "medical emergency" and "medical try law" and "regional law." Cheng-yu wu in the studies of medical behavior fault judgement, the article points out that the physician in specific health behaviors, should be at least as circumstances have a "medium level", it includes the professional, title, regional, habits and other factors to consider, "medium doctor" is not a specific entity, the judge should both doctors according to the situation that physicians should comply with the duty of care of boundary [2]. In the theory of medical treatment fault presumption of principles and standards, the article also puts forward physicians note standards include medical level, medical technology and medical behavior standard.

The duty of care is highly attention obligation, different from the general duty of care.In the general duty of care, the offender as long as do generally reasonable care, don't need to care and focus the attention of the state of mind to clear their behavior of the consequences.Person just from the rational level to control his behavior, avoid their actions bring endless harm to others.General taxpayers pay attention to the loss caused by breach of duty of care has remedial, such as the trustee 
not strictly examine the contents of the contract a duty of care, jeopardizing the interests of the principal, the trustee can make up for the client suffers economic losses.But doctors duty of care is because the identity of the physician and the characteristics of medical activities and needs highly cautious and careful, physicians should not only have the general rational person's mind, more need to make a reasonable choice of medical behavior.Physicians need to use solid medical professional knowledge and skilled professional skills, to heal the wounded and rescue the dying, save people's lives or professional spirit, the implementation of prudent and careful all have an impact on life and health of patients with medical practice.In the medical activity, the physician will be more dangerous than the common attention obligation, and the danger is more dangerous than general activity in the serious and urgent.Medical risk is not remedial, severity, light body for medical treatment fault behavior suffer more pain, or make the lives of patients.

Doctor duty of care is not limited to medical laws and regulations and the requirements of medical service contract, and the requirements of the professional ethics. The specific content of the notice obligation cannot be negotiated by both doctors and can only be determined by doctors in medical activities.Medical activities determines the content of the physician should take on more high degree of attention.Physicians to take emergency measures to rescue patients need reasonable, shall complete may not refuse treatment patients need law to a duty of care.At the same time, the judgement standards of other experts obligation to execute the effect is as a result, such as certified public accountants must provide accurate and reliable financial information, the lawyer must ensure the result of the parties get a fair judgment.But limited to the limits of medical technology development, physician obligation to execute the effect of the gauge is a means and process, rather than the result.

Doctors do not guarantee the performance of the obligations the cause of the patient completely eliminate, such as when a patient is obtained between the waist dish outstanding disease by doctor make a diagnosis and give treatment to eliminate completely, can only ease the deterioration. Other experts, can be found, a duty of care to protect the trust inanimate health security interests, doctors' duty of care to protect is trust the life and health security interests. Physicians for the implementation of the duty of care to reflect periodically.In the process of the physician to perform the duty of care, according to the specific medical doctors are engaged in activities, physicians should first cognitive the specific risks and matters needing attention in the medical activity, with his has the ability and knowledge, earnestly implement good diagnostic and therapeutic procedures. Physicians in the field of guarantee their ability to comply with professional work under the premise of minimum requirements, have not appeared under certain medical level [3]. This requires physicians with basic knowledge and basic skills of career in the medical profession, with the simplest of physicians to fulfill a duty of care.Second is good for medical purposes, doctors should take myself to share information and questions in clinical activities, use of consultation, ask for instructions, referral medical auxiliary means, such as the duty of care to establish foundation of other contents for the realization of the case of emergency. Finally is in the process of implementation of medical practice, physicians should be according to the actual circumstances of the patients, time to do a physician's attention obligation specific circumstances. At this point to the attention of the object is driven by the content of the medical treatment activity and doctor-patient set by both sides of the actual situation.

A duty of care only physicians to determine whether physician for medical treatment fault and medical behavior is illegal.But because of the diversity and complexity of specific medical activities, with the development of medicine, the evolution of the doctor-patient relationship, and the improvement of the legislation technology, may produce new duty of care. The specific requirements of the physician's duty of care is not the same. $\mathrm{N}$ nail a simple frame with established norms physician the content of the duty of care is difficult to adapt to the reality.The doctor should be a duty of care is a dynamic, with the development of age and medical progress will continue to expand its denotation.In the judicial process, to balance the interests of both doctors judge according to fair legal theory to interpret the content of the duty of care to physicians. To be sure, the discretion of the judge is not on the attached additional obligation, as doctors enter the health care industry and have certain professional ability, have know they should fulfill its obligations to the attention of the condition of 
reasonable boundary, it is the overall quality requirement of the workers in the health care industry.

The notice obligation origins has two kinds: one kind is formal law duty of care, a kind of informal method includes the duty of care. The former is the duty of care of law norms, it contains the laws and administrative regulations and department rules on the notice obligation;The latter refers to the law norms have not been a duty of care, but it is to avoid the occurrence of medical risk, physicians must comply with the duty of care in medical treatment activity.Article 5 of the regulations on the medical accident treatment in our country: "medical institution and its medical staff in medical treatment activity, must strictly abide by the medical and health management regulations of laws, administrative regulations, department and diagnosis and treatment to nurse standard, conventional, abide by the medical services, professional ethics."

The physician should be concerned about, love, respect patients, protect the privacy of patients, doctors found that patients with suspected damage events or wrongful death, report to the relevant departments shall, in accordance with the relevant regulations. In critically ill patients with emergency situations, doctors should adopt emergency measures to make a diagnosis and give treatment, and shall not refuse to give emergency treatment. Physician for medical treatment, prevention or health care measures, signing the relevant medical certificates, must be examined in person, investigation, and work out medical documents in a timely manner in accordance with the relevant provisions, shall not conceal, forge or destroy medical documents and relevant materials. No issue has anything to do with their own scopes of business or is not consistent with what category of medical documents.

Regular medical behavior should comply with the procedures and precautions that should be paid attention to, for the purpose of specific provision of a disease clinical manifestation, auxiliary examination, treatment, treatment principle, the principles, indications, contraindications, drug treatment effect evaluation and so on, these provisions are doctors must abide by the medical attention duty. At present, the health department has not yet issued a unified application in the medical technology in the process of practice. In accordance with the relevant theoretical explanation, medical accident appraisal practice and judicial practice, diagnosis and treatment technology operation specification includes the following: the relevant departments of the state council (the ministry of health, the state administration of traditional Chinese medicine, the state food and drug administration) for the diagnosis and treatment technology of operation specification, such as the ministry of health of the technical specification for clinical blood transfusion, the commonly used conventional family planning technology. The army formulated by the administrative department of public health diagnosis and technical operation specification, such as the general logistics department of the ministry of health to write the technical operation of conventional medical care and series complete works ". The national pharmacopoeia committee formulated by the drug specifications, such as the "pharmacopoeia of the People's Republic of China" and "pharmacopoeia of the People's Republic of China, clinical medication notice a chemical medicines and biological products volume". General medical colleges teaching and medical encyclopedia, such as teaching material of "internal medicine" and "surgery" and "Chinese medical encyclopedia" each volume. Diagnosis standard specifies the technical medical procedures, can say it to specific risks and matters needing attention in medical treatment activity standardization of summary, in order to make the medical behavior predictability and controllability [4]. Generally speaking, a sound diagnosis and specification represents the progress of the medical career, shows that man's ability to overcome the disease. As experts, doctors have carefully according to the process of diagnosis and treatment norms and conventional practice of obligation, the obligation is the most basic duty of care, is the reliable guarantee for the patient.

In the form of medical service contract is written and formatted, the prescriptions of the body of the contract is square, object to the rights and obligations of both parties to a patient objects: medical behavior, medical service behavior, both doctors content is based on medical contract or legal provisions, and enjoy the rights and obligations. Medical service contract is aimed at treating patients, patient's life and body is not to say the medical service contract subject matter, but the patient is freely give their life and body temporary transfer to the prescriptions and empirical, ShiCuoXing by its 
treatment, and make it not aggravating illness or increase the pain for diagnosis and treatment. $\mathrm{N}$ the contents stipulated in the seal between doctor and patient can include the price of treatment, treatment, treatment period, tells the service and so on, as long as it is consensual real intention, can be protected by law [5]. Although medical technology operation not specifically agreed between the parties in the patient but the content of the medical service contract can be more clearly request doctors must indeed provides patients with careful responsible, consistent with the medical technology level of development of medical service [6]. Because "people at risk, not suggest that others should be negligent act or say they don't want others to do their attention." the contract essentially improved the effect of physicians for the implementation of the duty of care, but that doesn't mean medical effects and the patient expectations is a physician wrong, as long as the corresponding duty of care physicians, medical effect of the average medical technology level.

\section{Summary}

Great changes have taken place in recent years, the doctor-patient relationship, doctor authority fall and rise the autonomy of patients. Increasingly strained doctor-patient relationship and medical disputes increase gradually, it become the main factors restricting the construction of a harmonious doctor-patient relationship. In an increasingly tense doctor-patient relationship, patients tend to stand on a kind of what kind of doctor or medical, few researchers have focused on, and the attitude to the clear to reduce medical disputes, the construction of a harmonious doctor-patient relationship is of great importance and value. Attitude is a kind of orientation of the individual state of psychological preparation, social psychologists now prevailing in three respects: cognition, emotion and behavior defined manner. Integrated foreign related literature, can think, patients for doctors in patients with medical attitude means yes or no cognitive evaluation, emotional reaction and behavior when go to a doctor.

\section{References}

[1] Clark N.Similarities and Differences between Scientific and Tech-nological Paradigms. Futures . 1987

[2] Osborne v.Montgomery. 203 Wis.223 234,N.W. 372 . 1931

[3] Andre Tunc.International Encyclopedia of Comparative Law Vol.4,Torts, Introduction. 1974

[4] J Stapleton.Duty of Care:Peripheral Parties and Alternative Opportunities for Deterrence. L.Q.R . 1995

[5] L Alexander.Causation and Corrective Justice:Does Tort Law Make Sense?‘. Law\&Phil1 . 1987

[6] Ying Dang,Jiangxiao Zhang,Ruiyu Li,Yue Li,Meng Li,Jun Qiao Guo,Jingtao Sun. The Research on the Privacy Protection of Hospital Patient in the Age of Internet Plus[A]. Advances in Social Science, Education and Humanities Research ( Volume 70) [C]. 2016 